\title{
A PRESCRIÇÃO RACIONAL EM MEDICINA VETERINÁRIA E OS NOMES DOS FÁRMACOS DOS MEDICAMENTOS ALOPÁTICOS
}

\author{
RATIONAL PRESCRIPTION IN VETERINARY MEDICINE AND THE NAMES \\ OF ACTIVE SUBSTANCES OF ALOPATIC MEDICINES
}

\author{
- REVISÃo BIBLIOGRÁFICA -
}

\author{
Augusto Langeloh ${ }^{1}$
}

\section{RESUMO}

Este artigo revisa a questão da prescrição dos medicamentos, no exercício da clínica veterinária, ressaltando as dificuldades e/ou problemas que podem resultar da existência de diversos nomes atribuidos a um mesmo fármaco. Os fármacos tem várias denominaçð̃es: o nome quimico, os nomes genéricos $e$ o nome comercial. Cada fármaco pode estar presente em mais de uma especialidade farmacêutica produzida pelas diversas indústrias farmacêuticas e recebe uma denominação comercial distinta. Uma portaria ministerial aprovou as Denominações Comuns Brasileiras baseadas na terminologia recomendada pela Organização Mundial da Saúde para o nome genérico dos fármacos, mas parte da indústria farmacêutica de medicamentos de uso médico veterinário ainda não emprega a denominação recomendada em seus produtos. A multipligidade resultante cria dificuldades e pode induzir o médico-veterinário a erros no momento da prescrição ou utilização de medicamentos além de obrigá-lo a freqüentes consultas a compêndios a procura da sinonimia mais conhecida para exercer uma terapêutica racional. Por outro lado, o intercâmbio comercial decorrente do acordo Mercosul, se incluir a comercialização de medicamentos veterinários, pode gerar dificuldades adicionais caso não sejam adotadas normas uniformes na denominação das substâncias ativas entre os paises signatários do acordo.

Palavras-chave: nome genérico, nome quimico, nome comercial, nomes de fármacos, prescrição, receita.
SUMMARY

\begin{abstract}
Drugs can be designed by their chemical name, by their generic (or non proprietary) names and by the commercial (or proprietary) name. Drugs can be marketed by more than one company and can have several proprietary or trademark names. In Brazil pharmaceutical companies which produce veterinary drugs use different chemical or generic names to designe the active drug of medicines. This clearly create difficulties for veterinarians inducing errors and misuses. Medicines for human use on the other hand have a official generic (non-proprietary) name known as Denominações Comuns Brasileiras (Brazilian Common Denomination) which were adopted in accordance with the World Health Organization recomendations. It is stressed that Brazilian Oficial non-proprietary names should also be adopted by manufacturers of veterinary drugs. This would either help to eliminate the necessity for memorizing multiple chemical and generic drug names as been as reduce the possibility of misunderstanding and errors. If veterinary medicines would be marketed in various South-American countries, as a result from the Mercosul comercial agreement, the existence of many names for the same drug can result in an even more confuse situation. This article deals with this problem and calls for attention for the uniformity of drug names.
\end{abstract}

Key words: generic name, chemical name, proprietary name, drug name, prescription.

'Médico Veterinário, Mestre e Doutor em Farmacologia, Professor Adjunto do Departamento de Farmacologia do Instituto de Biociências da UFRGS, Rua Professor Sarmento Leite, 500/202, 90046-900 - Porto Alegre, RS. 


\section{INTRODUÇÃO}

Prescrever racionalmente em MedicinaVeterinária significa eleger o(s) melhor(es) medicamento(s) para prevenir ou combater a doença de um animal ou grupo de animais, levando em conta diagnóstico, prognóstico, relação custo/benefício, as expectativas do criador ou proprietário e, quando for o caso, resguardando os interesses do consumidor dos produtos de origem animal. Prescrever corretamente é uma tarefa difícil que, após o estabelecimento do diagnóstico, requer conhecimento do "arsenal" terapêutico, atualização constante através de consulta a fontes bibliográficas confiáveis, e, sobretudo, responsabilidade (MILLER, 1977; PUGH, 1991; DAVIES, 1995; LANGELOH, 1995). Um levantamento realizado na $26^{a}$ edição do Compêndio Veterinário (ANDREI, 1993 ) revelou que existem 798 especialidades farmacêuticas distribuidas em 6 (seis) dos principais grupos farmacológicos de importância médico-veterinária, compreendendo: a) anti-helmínticos, b) quimioterápicos e antibióticos, c) ectoparasiticidas, d) analgésico/antitérmicos/anti-inflamatórios (AINE), e) tranquilizantes/anestésicos e f) fármacos com ação hormonal. Neste número não se incluem, portanto, as especialidades farmacêuticas integradas exclusivamente por vitaminas, oligoelementos e sais minerais, os biológicos e outras categorias. A estas especialidades de uso médico-veterinário podem se somar, para inúmeras situações práticas na clínica Médico Veterinária, em uso "extra-rótulo"(a), um número certamente elevado de medicamentos da linha "humana", discriminados na literatura pertinente (ANDREI, 1989; DEF 93/94). A eleição de uma (ou mais) destas especialidades farmacêuticas envolve vários parâmetros, um dos quais é a escolha do melhor fármaco, definido como a substância ativa do medicamento alopático, eficaz para o caso em pauta. Desta escolha e da implementação do esquema terapêutico correto depende o sucesso do tratamento. Na prescrição, ao invés do nome genérico, prefere-se utilizar o nome comercial da especialidade terapêutica, preferência esta que parece ser internacional (BRITISH VETERINARY ASSOCIATION, 1991; DOUST, 1994). Neste caso o estabelecimento comercial, seja farmácia, drogaria ou outro (casa agrícola, por ex.), só pode vender o medicamento prescrito, isto é, não pode substituí-lo por outro sem consulta e consentimento do profissional que o prescreveu. Em medicina, entretanto, tem havido esforço oficial no sentido de incentivar a prescrição pelo nome genérico da substância ativa, com objetivo de reduzir os custos do tratamento, como se pode inferir do
Decreto $\mathrm{n}^{0} 793$ de 05.04 .93 (BRASIL, 1993a). Nestes casos a escolha da especialidade farmacêutica é delegada para o farmacêutico em atividade no estabelecimento escolhido pelo consumidor. Esta prática pode ser adotada mais facilmente em ambientes hospitalares pelos profissionais que nele atuam. Em Medicina Veterinária a implementação de procedimento similar seria dificultada ou mesmo inviabilizada pela diversidade de nomenclatura genérica atribuida a um mesmo fármaco como veremos no decorrer deste trabalho.

Este artigo procura demonstrar que, além dos conhecimentos técnicos mais diretamente relacionados com o diagnóstico e dos conhecimentos de farmacologia e terapêutica, também é necessário conhecer os nomes químicos e genéricos dos fármacos e seus sinônimos para exercitar uma prescrição racional.

\section{Os nomes dos fármacos}

Os fármacos podem ser conhecidos $\mathrm{e}$ identificados através de várias denominações: o nome químico, o nome genérico (que podem ser vários) e 0 nome comercial (PUGH, 1991).

\section{Nome químico}

O nome químico seguramente é a mais precisa forma de denominar um fármaco pois enumera os seus constituintes, sua função química, posição, seqüência e relacionamento na estrutura molecular. Neste nome também deve estar incluído, se presente, a propriedade da substância, em solução, desviar a luz polarizada através da inclusão de um " $l$ " ou $(-)$ se levo ou de um " $d$ " ou $(+)$ se dextrorotatória, conforme o desvio da luz seja para a esquerda ou direita respectivamente. Esta é uma propriedade física de suma importância pois inúmeros fármacos só são eficazes farmacológicamente em uma dessas formas por exemplo sómente os isômeros levógiros do cloranfenicol e tetramisol são ativos. Um dado fármaco pode ter mais de um nome químico, como exemplificado com o tetramisol e o triclorfono na Tabela 1. Em geral, quanto mais complexa a estrutura química, maior a possibilidade de ter vários nomes químicos. Isto se deve a que os integrantes da molécula da substância em questão podem ser enumerados em ordem diferente ou, mesmo, parcialmente aglutinados em uma outra molécula conhecida cujo nome passa a fazer parte da nova substância. Apesar da sua "precisão" o nome químico nem sempre pode ou é utilizado. Por outro lado, mesmo que fosse, a perfeita compreensão da 
estrutura molecular a partir desta denominação fica limitada aos químicos e, às vezes, até restrita aos especialistas de um determinado ramo da química. A partir do nome químico também é difícil estabelecer a que grupo farmacológico pertencem muitas substâncias ativas. O nome químico deve ser grafado com inicial minúscula.

\section{Código alfa-numérico}

Nas fases preliminares de desenvolvimento e investigação de uma substância com potencialidade para se tornar um fármaco de interesse terapêutico importante, o laboratório envolvido costuma utilizar um código alfa-numérico (MOREAU, 1985). Esta sigla identifica esta substância e impede aos eventuais concorrentes de copiá-la pura e simplesmente, o que decerto seria facilitado se, nesta fase, fosse divulgado seu nome químico. Habitualmente a sigla é constituida de iniciais retiradas do próprio nome do laboratório ou indústria que desenvolve as investigações. O nome Bayva-1470 foi utilizado pela Bayer nos testes pré-clínicos e clínicos para a xilazina, hoje comercializado com o nome de Rompun $\circledast$. Algumas vezes o fármaco acaba se tornando conhecido mais por este número do que por qualquer outra denomina-ção. É o caso de dois quimioterá-picos, o CGA72662 correspondente a ciromazina e o NF180 correspon-dente a furazolidona, entre as especialidades de uso Médico veterinário. O emprego deste "nome" alfa-numérico, nas bulas, deve ser totalmente desestimulado ou mesmo proibido. As denomina-ções comuns brasileiras, vide abaixo, não incluem nenhuma denominação desta natureza.

\section{Nome(s) genérico(s)}

(c)

Neguvon

Antihelmin

ester dimetílico do ácido (2,2,2-tricloro-1-hidroxietil) fosfônico

0,0-dimetil-1-hidroxi-2,2,2-tricloroetilfosfonato

Ciclosom

Revermin

Vectrin Solução Injetável

(a) Ambas as informaçð̃es foram obtidas por consulta ao Compêndio Veterinário (ANDREI, 1993). O critério para escolher e incluir as especialidades farmacêuticas citadas como exemplo nesta Tabela foi aleatório;

(b) Esta denominação para o princípio ativo não pode ser confirmada em nenhum dos compêndios de nomes químicos consultados;

(c) Nenhuma especialidade farmacêutica de uso veterinário faz uso da DCB para esta substância ativa.

Em lugar do nome químico, muito complexo e/ou extenso, e do código alfa-numérico que nada informa sobre a estrutura ou atividade farmacológica do fármaco, aparecem os nomes genéricos. Estes podem ser comparados a "apelidos", pois são atribuídos em função de características químicas o que se reflete nas propriedades farmacológicas gerais. Algumas substâncias, entretanto, podem apresentar propriedades farmacológicas "únicas". Ex. o têrmo "penicilinas inclui a

Ciência Rural, v. 26, n. 2, 1996. 
benzilpenicilina (e seus vários sais) mais a ampicilina, carbenicilina e todas as outras que apresentam em comum o ácido-6-aminopenicilânico (6-APA). Esta molécula central, 6-APA, confere importantes propriedades comuns a este grupo de substâncias, mas cada um dos fármacos citados se caracteriza por propriedades farmacodinâmicas e farmacocinéticas exclusivas (e.g. a benzilpenicilina só é eficaz contra bactérias Gram positivas, a ampicilina tem eficácia contra bactérias Gram positivas e Gram negativas, etc. etc.). Outros exemplos de nomes gené-ricos que reunem vários representantes compreendem: aminoglicosídeos, barbitúricos, benzimidazóis, benzodiazepínicos, catecolaminas, sulfonamidas, etc.

O nome genérico pode ser específico, referindo-se a uma substância química determinada, como por exemplos benzilpenicilina, fenobarbital, cloranfenicol, diazepam e tiabendazol. A partir destas substâncias também pode-se obter sais distintos, que se diferenciam entre si por alguma(s) propriedade(s) farmacológica(s). Exemplificando: benzilpenicilina sódica; benzilpenicilina benzatínica; fenobarbital sódico; palmitato de cloranfenicol e o succinato de cloranfenicol, etc.

O nome genérico adotado para um fármaco pode ser diferente em distintos países: a noradrenalina (denominação européia) é a norepinefrina nos EEUU e no Brasil. A penicilina $G$ (denominação inglesa) é, no Brasil, a benzilpenicilina.

No passado, antes do estabelecimento de normas internacionais, um fármaco desenvolvido em laboratórios distintos, poderia receber de cada indústria um "apelido" próprio e diferente. Como resultado, independente do(s) nome(s) químico(s), um fármaco pode ter várias denominações genéricas (Tabelas $1 \mathrm{e}$ 2). Alguns laboratórios e/ou empresas produtoras de subtâncias químicas registraram e empregam o "apelido" que atribuiram a um dado fármaco por eles desenvolvido. Por exemplo Vapona é a denominação conferida ao diclorvos pela Shell (BUDAVARI et al. 1990). Esta situação, quase caótica, obrigou a elaboração e adoção de normas visando regular, uniformizar e facilitar a criação de nomes genéricos. O nome genérico das substâncias ativas deve ser grafado com inicial minúscula.

\section{Uniformização dos nomes genéricos}

A existência de tantas e tão diversas denominações para um mesmo fármaco provocaram a adoção de várias medidas, destacando-se: (a) o estabelecimento de normas internacionais para fixar o nome das substâncias ativas (MOREAU 1985;
KOROLKOVAS, 1988); (b) a recomendação de emprego de nomes genéricos oficiais também denominados de "nomes não propriedade" (non proprietary names) (KOROLKOVAS, 1988 e 1994; PUGH, 1991; DAVIES, 1995 ) e (c) a elaboração de dicionários com as sinonímias das substâncias químicas ( BUDAVARI et al. 1990). Os catálogos do Chemical Abstracts, o Index Merck, as Farmacopéias e as recomendações da OMS, entre outros, constituem exemplos do esforço para correlacionar as diferentes denominações atribuidas às substâncias químicas e/ou padronizá-lo.

Tabela 2. Sinonímia de nomes genéricos de alguns fármacos em consequência dos quais podem surgir dificuldades e ou confusão durante a prescrição porque a Denominação Comum Brasileira não é adotada por todas as especialidades farmacêuticas de uso veterinário existentes.

Denominação Comum Brasileira sinônimos encontrados

$\begin{aligned} & \begin{array}{r}\text { benzilpenicilina } \\ \text { colistina }\end{array} \text { penicilina } \mathrm{G} \\ & \text { éter etílico } \text { polimixina } \mathrm{E} \\ & \text { éter anestésico; éter } \\ & \text { sulfúrico } \\ & \text { guaifenesina } \text { guaiacolato de glice- } \\ & \text { rila; éter glicerilguaia- } \\ & \text { cólico } \\ & \text { lidocaína } \text { xilocaína } \\ & \text { norepinefrina } \text { noradrenalina; I arte- } \\ & \text { renol; } \\ & \text { paromomicina } \text { aminosidina } \\ & \text { procaína } \text { novocaína } \\ & \text { retinol } \text { Vitamina } \mathrm{A} \\ & \text { tocoferol } \text { Vitamina } \mathrm{E} \\ & \text { triclosano } \text { Irgasan DP300@ } \\ &\end{aligned}$

No Brasil, a Portaria ministerial $\mathrm{n}^{\circ} 971$ aprovou relação contendo a Denominação Comum Brasileira (DCB) para os fármacos, fundamentada na terminologia recomendada pela Organização Mundial da Saúde (OMS) procurando operacionalizar uma uniformização (BRASIL, 1993b). Esta medida porém não resolveu ainda os problemas existentes com os fármacos presentes em especialidades farmacêuticas de uso Médico veterinário (Tabela 1). 
Para facilitar o reconhecimento da classe ou do efeito farmacológico das substâncias ativas são usados prefixos ou sufixos iguais (KOROLKOVAS, 1988 e 1993). Exemplificando: a) terminam em "icilina" os antimicrobianos que tem ação semelhante à da penicilina (ampicilina, carbenicilina, etc.); terminam em "fos" ou "vos" os ectoparasiticidas a base de organofosforados (clorfenvinfos e diclorvos); terminam em "olol" os bloqueadores beta-adrenérgicos (propranolol, atenolol, etc.); começam com "cef" os antimicrobianos aparentados com as cefalosporinas (ex. cefaclor, cefaloridina, etc.), e assim por diante. Mas há exceções que podem causar sérias confusões como o organofosforado metrifonato (Tabela 1), cuja denominação correta é "triclorfono", o antibiótico aminosidina (um aminoglicosídeo também conhecido por paromomicina) entre outros que obrigam a consulta aos compêndios, à procura da sinonímia mais conhecida.

Os catálogos de sinonímia química são dinâmicos, pois a cada ano novos fármacos são acrescentados ao arsenal terapêutico. A consulta a estes compêndios pelo Médico veterinário, a cada troca de medicamento, não é prática. A solução, aparentemente muito mais simples, passa pela uniformização da denominação genérica utilizada para cada fármaco. Todas e cada especialidade farmacêutica com o mesmo fármaco devem empregar a mesma denominação ao identificar o(s) seu(s) constituinte(s) ativo(s) principalmente dentro de um mesmo país. Esta é uma possibilidade viável no Brasil onde todos os medicamentos de uso Médico veterinário necessitam de registro no Serviço de Controle de Produtos Veterinários do Departamento de Defesa Animal da Secretaria de Defesa Agropecuária do Ministério da Agricultura, do Abastecimento e da Reforma Agrária de acordo com o Regulamento de Fiscalização de Produtos de Uso Veterinário anexo ao Decreto 1.662 (BRASIL, 1995) que veio substituir o Decreto 64.499 de 14.05.1969. A uniformização deve ser procedida pela adoção da denominação comum brasileira (acima referida). Para os fármacos que ainda não tenham uma denominação comum brasileira, isto é, ainda não foram incluídos na Portaria 971 (BRASIL, 1993b), deve ser usado, provisoriamente, o nome proposto pela OMS ou, na falta deste, o da International Nonproprietary Names (INN) adotando-se a DCB assim que o nome for escolhido e divulgado. Estão previstas atualizações anuais das listas da DCB. Em especialidades farmacêuticas médico veterinárias é freqüente a introdução de substâncias ativas que ainda não foram incluidas na DCB e/ou que não são empregadas em especialidades destinadas ao uso humano (Tabela 3 ).
Tabela 3. Exemplos de fármacos presentes em especialidades farmacêuticas de uso médico veterinário não incluídas na Portaria 971 que lista a Denominação Comum Brasileira (BRASIL, 1993b) e sua principal indicação em terapêutica veterinária.

\begin{aligned} \hline Fármaco & \multicolumn{1}{c}{ Uso terapêutico } \\ \hline clorfenvinfos ou Supona $\left.{ }^{\circledR}\right) & \begin{array}{l}\text { organofosforado usado como } \\ \text { endo e ectoparasiticida } \\ \text { desinfetante } \\ \text { dicloroisocianuratosforado usado como } \\ \text { diclorfos ou DDVP } \\ \text { endo e ectoparasiticida } \\ \text { derivado do fenol usado como } \\ \text { anti-helmíntico } \\ \text { antibiótico antibacteriano e } \\ \text { aditivo alimentar } \\ \text { prostaglandina usada na sin- } \\ \text { cronização do estro } \\ \text { prostaglandina usada na sin- } \\ \text { cronização do estro } \\ \text { antibiótico com propriedades } \\ \text { anti-helmínticas } \\ \text { pré-benzilpenicilina (antimi- } \\ \text { crobiano) }\end{array} \\$ metilcarboprost & penetamato \\ milbemicina - oxima & \end{aligned}

\section{Nome comercial}

O nome comercial é uma denominação de fantasia atribuido às especialidades farmacêuticas que veiculan o(s) fármaco(s) previamente preparado pela indústria. É um nome registrado (KOROLKOVAS, 1988 e 1993; BUDAVARI et al. 1990). Salvo exceções ou coincidências, não guarda qualquer compromisso ou relação com as propriedades químicas ou farmacológicas da substância ativa. Além disso, muitas especialidades farmacêuticas contém mais de um fármaco apesar das recomendações em contrário em órgãos reguladores, e.g. Portaria 159 (BRASIL, 1992) e do fato da terapêutica racional preferir a administração de monopreparados (MILLER, 1977; BRITISH VETERINARY ASSOCIATION, 1991; PUGH, 1991; DOUST, 1994; DAVIES, 1995). Na Tabela 1 foram incluidos, a título de exemplo, o nome comercial de especialidade farmacêutica de uso Médico veterinário que, na bula, utiliza o nome químico ou genérico com a qual está relacionada. O nome comercial deve ser grafado com inicial maiúscula.

\section{Exemplificando com problemas do dia-a-dia}

Para ilustrar alguns possiveis problemas decorrentes da falta de uniformidade na denominação 
de fármacos freqüentemente empregados em terapêutica médico veterinária foram reunidos exemplos representativos na Tabela 1. Com relação ao cloranfenicol foram encontradas três formas diferentes de se referir a esta substância ativa, enquanto que para o organofosforado diclorfos foram encontradas 4 denominações distintas. O clínico desinformado ou desatento que, por causa de insucesso, eventualmente proceda substituições no curso de um tratamento, entre as especialidades farmacêuticas contendo cloranfenicol ou diclorfos, reunidas na Tabela 1 para exemplificação, poderá se frustar: há uma grande probabilidade de que a substituição não resulte em melhora do quadro clínico ou controle da doença. A troca entre medicamentos contendo o mesmo fármaco, ainda que com denominações diferentes na bula, só terá sucesso se o primeiro medicamento usado era deficiente quanto a concentração preconizada na bula ou quanto a liberação do fármaco para absorção, se estava com prazo de validade vencido, etc. e a segunda especialidade farmacêutica preencher os requisitos mínimos assegurando uma biodisponibilidade adequada.

Problemas de mesma natureza podem ocorrer com os demais exemplos que integram a Tabela 1. Apenas um será usado a título de ilustração: assim a troca ou mudança entre os produtos Neguvon, Antihelmin, Cicloson e Revermin não trará nenhum benefício se a causa do tratamento ocorre por resistência dos helmintos, hipersensibilidade tóxica ou alérgica do paciente ao triclorfono. Todos são à base do mesmo fármaco, um organofosforado, portanto tem em comum o mesmo espectro e demais características farmacológicas, ainda que nas respectivas bulas a composição possa revelar quatro nomes distintos, sugerindo que sejam diferentes. A única forma de saber que os quatro nomes se referem a mesma substância ativa é a consulta ao Index Merck, Chemical Abstracts ou equivalentes ou, ainda, a um bom livro texto de Farmacologia... Há casos em que a consulta a estes compêndios tampouco ajuda: por exemplo a substância ativa da especialidade farmacêutica Leivamisole, dado como leivamisol, sinonímia que não aparece em nenhum compêndio consultado.

\section{CONCLUSÃO}

Pretendeu-se neste artigo chamar atenção dos Médicos veterinários clínicos para uma questão relevante e adicional aos problemas naturalmente já existentes para elaborar uma prescrição e na instituição de um tratamento (LANGELOH, 1995). Especifi- camente relacionado com a ausência de uniformidade na denominação dos fármacos das centenas de especialidades farmacêuticas disponíveis para escolha pelo profissional. Esta falha pode causar frustrações terapêuticas e, mesmo, suscitar erros médicos. O Médico veterinário deve estar consciente da possibilidade deste problema e procurar formar-se e informar-se adequadamente cada vez que incluir uma nova especialidade farmacêutica ao seu arsenal e toda vez que um esquema posológico, seguido a risca, não resultar no sucesso esperado. A situação poderá ficar ainda mais confusa diante da iminente implementação de trocas comerciais entre os países integrantes do acordo MERCOSUL, se os medicamentos de uso Médico veterinário forem incluídos no intercâmbio (PEREIRA, 1993). Deve-se estar consciente que este intercâmbio pode introduzir ainda mais variáveis na já complicada situação da terminologia dos fármacos. Para evitá-las caberiam reuniões entre os responsáveis e representantes dos órgãos oficiais que cuidam do registro dos medicamentos de uso Médico veterinário, procurando uniformizar as suas denominações. Este aspecto não foi contemplado explicitamente no documento gerado pelas autoridades dos países envolvidos, o chamado Marco Regulatório Para os Produtos Veterinários. Normas elaboradas com o objetivo de uniformizar as denominações dos fármacos entre os signatários do acordo MERCOSUL deveriam acolher as recomendações dos organismos internacionais, e.g. OMS, as quais já foram adotadas no Brasil na elaboração das Denominações Comuns Brasileiras.

\section{AGRADECIMENTOS}

O autor agradece aos professores Dr. J.R.B.de Mello do Departamento de Farmacologia e Dr.E.P.Schenkel do Departamento de Produção de Matéria Prima, ambos da UFRGS pela análise e revisão deste artigo.

\section{FONTES}

a - Entende-se por uso extra-rótulo o emprego de uma especialidade farmacêutica para uma finalidade diferente das especificadas nas indicações da bula ou, ainda, para uma espécie diferente da(s) indicada(s) na bula. Nestes casos o veterinário que prescreve é o único responsável pelas consequeências danosas eventualmente provocadas no paciente e/ou consumidor de produtos de origem animal.

\section{REFERÊNCIAS BIBLIOGRAFICAS}

ANDREI - Compêndio Médico. $29^{a}$ ed. São Paulo: Organização Andrei Editora Ltda, 1989. 822 p. 
ANDREI - Compêndio Veterinário. $26^{a}$ ed. São Paulo: Organização Andrei Editora Ltda , 1993. 683 p.

BRASIL, Ministério da Agricultura e Reforma Agrária. Portaria 159 de 19.06.1992, Diário Oficial da União. Sec I, p. 7942-3, 23.06.1992.

BRASIL, Decreto 793 de 05.04.1993. Diário Oficial da União. Sec.l. no 65. p. 4398, 6 abr 1993a.

BRASIL, Ministério da Saúde, Portaria 971 de 10.08.1993, Diário Oficial da União Sec I. n. 154, p 11745-77, 13 ago. 1993b.

BRASIL, Decreto 1662 de 06.10.95, Diário Oficial da União. In: Veterinária \& Zootecnia, no 8, p. 16, 1995.

BRITISH VETERINARY ASSOCIATION (BVA) - Prescribing of medicinal products by veterinary surgeons. Veterinary Record, v. 14: p. 245-8, 1991.

BUDAVARI, S., O'NEIL, M.J., SMITH, A. et al. INDEX MERCK, 11 th. ed. Rahway, New Jersey: Merck \& Co., 1990. $1606 \mathrm{p}$.

DAVIES, L.E. Drug Prescription Orders. In: RICHARD ADAMS, A. ed. Veterinary Pharmacology and Therapeutics. 7th. ed. Ames, lowa, USA: Iowa State University Press, 1995. Cap. 1. p. 1141-2.

DEF93/94 - Dicionário de Especialidades Farmacêuticas. São Paulo: Ed. Jornal Brasileiro de Medicina; 1993. 876 p.
DOUST, T. Regulation of veterinary chemicals in Australia. Australian Veterinary Journal, v. 71, p. 407-9, 1994.

KOROLKOVAS, A. Essentials of Medicinal Chemistry. London: Ed. John Wiley \& Sons Inc., 1988, p. 16-26.

KOROLKOVAS, A. Nomenclatura de Fármacos. Infarma, Brasilia, v. 2, p. 13-4, 1993.

KOROLKOVAS, A. Proposta para padronização das Denominaç̃es Comuns Brasileiras. Cadernos de Farmácia, Porto Alegre, v.10, p. 15-7, 1994.

LANGELOH, A. A prescrição de medicamentos e sua legislação aplicada a clínica veterinária. Arquivos da Faculdade de Veterinária da UFRGS, Porto Alegre, v. 24, p. 210-43, 1995

MILLER, L.C. Doctors, Drugs, and Names. Journal of the American Medical Association, v. 177, p. 97- 103, 1977.

MOREAU, R. The naming ceremony. World Health, v. 10, p. 8$9,1985$.

PEREIRA, M.S. O Mercosul e a indústria veterinária. Veterinária e Zootecnia. Porto Alegre, v. 1, p.4, 1993.

PUGH, D.M. Pharmacology and the veterinarian. In: BRANDER, G.C., PUGH, D. M., BYWATER, R.J. \& JENKINS, W.L. Veterinary applied Pharmacology \& Therapeutics. 5th ed. London: Baillière Tindall, 1991. Cap. 1. p. 3-8.

Ciência Rural, v. 26, n. 2, 1996. 